\title{
ON NUMERICALLY EFFECTIVE LOG CANONICAL DIVISORS
}

\author{
SHIGETAKA FUKUDA
}

Received 18 March 2001 and in revised form 9 October 2001

Let $(X, \Delta)$ be a 4-dimensional log variety which is proper over the field of complex numbers and with only divisorial log terminal singularities. The $\log$ canonical divisor $K_{X}+\Delta$ is semiample, if it is numerically effective (NEF) and the Iitaka dimension $\kappa\left(X, K_{X}+\Delta\right)$ is strictly positive. For the proof, we use Fujino's abundance theorem for semi-log canonical threefolds.

2000 Mathematics Subject Classification: 14E30, 14J35, 14C20.

1. Introduction. In this paper, every variety is proper over the field $\mathbb{C}$ of complex numbers. We follow the notation and terminology of [11].

Let $X$ be a normal algebraic variety and let $\Delta=\sum d_{i} \Delta_{i}$ be a $\mathbb{Q}$-divisor with $0 \leq d_{i} \leq 1$ on $X$ such that the log canonical divisor $K_{X}+\Delta$ is $\mathbb{Q}$-Cartier. We call $(X, \Delta)$ a log pair.

Let $D$ be a numerically effective (NEF) $\mathbb{Q}$-Cartier $\mathbb{Q}$-divisor on $X$. We define the numerical Iitaka dimension $v(X, D):=\max \left\{e ;\left(D^{e}, S\right)>0\right.$ for some subvariety $S$ of dimension $e$ on $X$ \}. The divisor $D$ is abundant if the Iitaka dimension $\kappa(X, D)$ equals $v(X, D)$. If, for some positive integer $m$, the divisor $m D$ is Cartier and the linear system $|m D|$ is free from base points, $D$ is said to be semiample.

For a birational morphism $f: Y \rightarrow X$ between normal algebraic varieties and for a divisor $E$ on $X$, the symbol $f_{*}^{-1} E$ expresses the strict transform of $E$ by $f$, and $f^{-1}(E)$ expresses the set-theoretical inverse image. A resolution $\mu: Y \rightarrow X$ is said to be a $\log$ resolution of the $\log$ pair $(X, \Delta)$ if the support of the divisor $\mu_{*}^{-1} \Delta+\sum\{E ; E$ is a $\mu$-exceptional prime divisor $\}$ is with only simple normal crossings. The log pair $(X, \Delta)$ is $\log$ terminal if there exists a log resolution $\mu: Y \rightarrow X$ such that $K_{Y}+\mu_{*}^{-1} \Delta=$ $\mu^{*}\left(K_{X}+\Delta\right)+\sum a_{i} E_{i}$ with $a_{i}>-1$. Moreover, if the exceptional locus $\operatorname{Exc}(\mu)$ consists of divisors, $(X, \Delta)$ is said to be divisorial log terminal (DLT). Szabó [16] proved that the notions of DLT and wklt in [15] are equivalent. In the case where $(X, \Delta)$ is log terminal and $\lfloor\Delta\rfloor=0$, we say that $(X, \Delta)$ is Kawamata log terminal (KLT).

We note that if $(X, \Delta)$ is KLT then it is DLT. In the Iitaka classification theory of open algebraic varieties, one embeds a smooth affine variety $U$ in some smooth projective variety $X$ such that $X \backslash U \operatorname{Supp}(\Delta)$, where $\Delta$ is a reduced simple normal crossing divisor, and studies the log pair $(X, \Delta)$. In this case $(X, \Delta)$ is not KLT but DLT. Moreover, it is known that we have to work allowing the $\mathbb{Q}$-factorial DLT singularities, to execute the log minimal model program for open algebraic varieties (see [9]). Therefore, it is valuable to extend theorems proved in the case of KLT pairs to the case of DLT pairs.

Now, concerning the log minimal model program, we review the following famous conjecture. 
LOG ABUNDANCE CONJECTURE 1.1 (cf. [10]). Assume that $X$ is projective and $(X, \Delta)$ is DLT. If $K_{X}+\Delta$ is NEF, then $K_{X}+\Delta$ is semiample.

This conjecture claims that the concept of "log minimal" (i.e., the log canonical divisor is NEF) should be not only numerical but also geometric. Kawamata [7] and Fujita [5] proved the conjecture in $\operatorname{dim} X=2$ and Keel, Matsuki, and McKernan [10] proved it in $\operatorname{dim} X=3$. (The assumption concerning singularities in their papers is that $(X, \Delta)$ is log canonical, which is more general than DLT.) Moreover, Fujino proved the following theorem.

THeOrem 1.2 (see [3, Theorem 3.1]). Assume that $(X, \Delta)$ is DLT and $\operatorname{dim} X=4$. If $K_{X}+\Delta$ is NEF and big, then $K_{X}+\Delta$ is semiample.

The following two theorems, due to Kawamata, are helpful to deal with the conjecture.

THEOrem 1.3 (see [8, Theorem 6.1]). Assume that $(X, \Delta)$ is KLT and $K_{X}+\Delta$ is NEF. If $K_{X}+\Delta$ is abundant, then it is semiample.

TheOrem 1.4 (see [8, Theorem 7.3], cf. [10, Lemma 5.6]). Assume that $(X, \Delta)$ is KLT and $K_{X}+\Delta$ is NEF. If $\kappa\left(X, K_{X}+\Delta\right)>0$ and the log minimal model and the log abundance conjectures hold in dimension $\operatorname{dim} X-\kappa\left(X, K_{X}+\Delta\right)$, then $K_{X}+\Delta$ is semiample.

In this paper, we try to generalize the above-mentioned theorems and obtain the following main theorem.

THEOREM 1.5. Assume that $(X, \Delta)$ is DLT and $\operatorname{dim} X=4$. If $K_{X}+\Delta$ is NEF and $\kappa\left(X, K_{X}+\Delta\right)>0$, then $K_{X}+\Delta$ is semiample.

We prove the main theorem (Theorem 1.5) along the lines in the proofs of Theorems 1.2 and 1.3, using Fujino's abundance theorem for semi-log canonical threefolds which are not necessarily irreducible. (For the definition of the concept "SDLT" appearing below, see Definition 2.7 in Section 2.)

THEOREM 1.6 (see [2]). Let $(S, \Theta)$ be an SDLT threefold. If $K_{S}+\Theta$ is NEF, then $K_{S}+\Theta$ is semiample.

REMARK 1.7. If the log minimal model and the log abundance conjectures hold in dimension less than or equal to $n-1$, and Theorem 1.6 holds in dimension $n-1$, then Theorem 1.5 holds in dimension $n$.

2. Preliminaries. In this section, we state notions and results needed in the proof of Theorem 1.5. The next two propositions are from the theories of the Kodaira-Iitaka dimension and the minimal model, respectively.

Proposition 2.1 (see [6, Theorem 10.3]). Let D be an effective divisor on a smooth variety $Y$. Suppose that the rational map $\Phi_{|D|}: Y \rightarrow Z$ is a morphism between algebraic varieties and that the rational function field $\operatorname{Rat}\left(\Phi_{|m D|}(Y)\right)$ is isomorphic to $\operatorname{Rat}(Z)$ for all positive integer $m$. Then $\operatorname{Rat}(Z)$ is algebraically closed in $\operatorname{Rat}(Y)$ and $\kappa\left(W,\left.D\right|_{W}\right)=0$ for a "general" fiber of $\Phi_{|D|}$. 
Proposition 2.2 (see [9, Section 5-1]). Assume that $\left(X_{l m}, \Delta_{l m}\right)$ is a log minimal model for a $\mathbb{Q}$-factorial, $D L T$ projective variety $(X, \Delta)$. Then, every common resolution $X \stackrel{g}{\longrightarrow} Y \stackrel{h}{\longrightarrow} X_{l m}$ satisfies the condition that

$$
K_{Y}+g_{*}^{-1} \Delta+E \geq g^{*}\left(K_{X}+\Delta\right) \geq h^{*}\left(K_{X_{l m}}+\Delta_{l m}\right)
$$

where $E$ is the reduced divisor composed of the $g$-exceptional prime divisors.

The following is a vanishing theorem of Kollár-type.

Theorem 2.3 (see [12, Theorem 10.13], [8, Theorem 3.2], [1, Section 3.5]). Let $f$ : $X \rightarrow Y$ be a surjective morphism from a smooth projective variety $X$ to a normal variety $Y$. Let $L$ be a divisor on $X$ and $D$ an effective divisor on $X$ such that $f(D) \neq Y$. Assume that $(X, \Delta)$ is $K L T$ and $L-D-\left(K_{X}+\Delta\right)$ is $\mathbb{Q}$-linearly equivalent to $f^{*} M$ where $M$ is a $N E F$ and big $\mathbb{Q}$-Cartier $\mathbb{Q}$-divisor on $Y$. Then the homomorphisms $H^{i}\left(X, O_{X}(L-D)\right) \rightarrow$ $H^{i}\left(X, \mathcal{O}_{X}(L)\right)$ are injective for all $i$.

When we work on the non-KLT locus $\lfloor\Delta\rfloor$ of a log terminal pair $(X, \Delta)$, we need the following lemma.

LEMMA 2.4 (cf. [6, Proposition 1.43]). Let $S$ be a reduced scheme and F an invertible sheaf on $S$. Then, the restriction map $H^{0}(S, \mathscr{F}) \rightarrow H^{0}(U, \mathscr{F})$ is injective for all open dense subset $U$ of $S$.

The following lemma is used to manage cases where Theorem 2.3 cannot be applied (see [10, Section 7]).

LEMMA 2.5 (cf. [4, Section 1.20]). Let $f: S \rightarrow Z$ be a surjective morphism between normal varieties and $H_{Z}$ a Cartier divisor on $Z$. If $f^{*} H_{Z}$ is semiample, then so is $H_{Z}$.

The set $\operatorname{Strata}(D)$ defined below is the set of non-KLT centers for a smooth pair $(Y, D)$.

DEFINITION 2.6. Let $D=\sum_{i=1}^{l} D_{i}$ be a reduced simple normal crossing divisor on a smooth variety $Y$. We set $\operatorname{Strata}(D):=\left\{\Gamma ; 1 \leq i_{1}<i_{2}<\cdots<i_{k} \leq l\right.$, $\Gamma$ is an irreducible component of $\left.D_{i_{1}} \cap D_{i_{2}} \cap \cdots \cap D_{i_{k}} \neq \varnothing\right\}$.

When we manage the non-KLT locus $\lfloor\Delta\rfloor$ of a DLT pair $(X, \Delta)$, we need the following notion.

Definition 2.7 (see [2, Definition 1.1]). Let $S$ be a reduced $S_{2}$ scheme which is pure $n$-dimensional and normal crossing in dimension 1 . Let $\Theta$ be an effective $\mathbb{Q}$-Weil divisor such that $K_{S}+\Theta$ is $\mathbb{Q}$-Cartier. Let $S=\bigcup S_{i}$ be the decomposition into irreducible components. The pair $(S, \Theta)$ is semi-divisorial $\log$ terminal $(S D L T)$ if $S_{i}$ is normal and $\left(S_{i},\left.\Theta\right|_{S_{i}}\right)$ is DLT for all $i$.

Proposition 2.8 (see [2, Remark 1.2.(3)], [15, Section 3.2.3], [13, Corollary 5.52]). If $(X, \Delta)$ is DLT, then $(\lfloor\Delta\rfloor, \operatorname{Diff}(\Delta-\lfloor\Delta\rfloor))$ is SDLT.

3. Proof of the main theorm (Theorem 1.5). The following proposition is used to imply the abundance of some log canonical divisor from its mobility. 
Proposition 3.1 (see [8, Theorem 7.3], [10, Lemma 5.6]). Let $(X, \Delta)$ be a variety with only log canonical singularities such that $K_{X}+\Delta$ is NEF and $\kappa\left(X, K_{X}+\Delta\right)>0$. If the log minimal model and the log abundance conjectures hold in dimension $\operatorname{dim} X-$ $\kappa\left(X, K_{X}+\Delta\right)$, then $\kappa\left(X, K_{X}+\Delta\right)=\nu\left(X, K_{X}+\Delta\right)$.

In the literature (see [8, Theorem 7.3]), this is proved for KLT pairs. However, the proof is valid for log canonical pairs also. Thus, in the proof below we note only the parts where we have to be careful in reading [8, Proof of Theorem 7.3].

Proof. (See [8, Proof of Theorem 7.3].) By Proposition 2.1, we have a diagram

$$
X \stackrel{\mu}{\longrightarrow} Y \stackrel{f}{\longrightarrow} Z
$$

with the following properties:

(a) $Y$ and $Z$ are smooth projective varieties. Moreover, $Y$ is a log resolution of $(X, \Delta)$;

(b) $\mu$ is birational and $f$ is surjective. The morphism $f$ satisfies that $\operatorname{dim} Z=\kappa(X$, $\left.K_{X}+\Delta\right)$ and $f_{*} O_{Y}=O_{Z}$

(c) $K_{Y}+\mu_{*}^{-1} \Delta+E=\mu^{*}\left(K_{X}+\Delta\right)+E_{\mu}$, where $E$ is the reduced divisor composed of the $\mu$-exceptional prime divisors and $E_{\mu}$ is an effective $\mathbb{Q}$-divisor;

(d) for a general fiber $W=Y_{z}$ of $f,\left.K_{Y}\right|_{W}=K_{W}$ and $\kappa\left(W, K_{W}+\left.\left(\mu_{*}^{-1} \Delta+E\right)\right|_{W}\right)=0$.

We note that $W$ is smooth and $\operatorname{Supp}\left(\left.\left(\mu_{*}^{-1} \Delta+E\right)\right|_{W}\right)$ is with only simple normal crossings.

We apply the log minimal model program to $\left(W,\left.\left(\mu_{*}^{-1} \Delta+E\right)\right|_{W}\right)$ and obtain a log minimal model $\left(W_{l m}, \Delta_{l m}\right)$, where $K_{W_{l m}}+\Delta_{l m} \sim_{\mathbb{Q}} 0$ from the log abundance. We consider a common resolution $W \stackrel{\rho}{\longleftarrow} W^{\prime} \stackrel{\sigma}{\longrightarrow} W_{l m}$ of $W$ and $W_{l m}$ such that $W^{\prime}$ is projective. From Proposition 2.2,

$$
\rho^{*}\left(K_{W}+\left.\left(\mu_{*}^{-1} \Delta+E\right)\right|_{W}\right)=\sigma^{*}\left(K_{W_{l m}}+\Delta_{l m}\right)+E_{\sigma} \sim_{\mathbb{Q}} E_{\sigma}
$$

for some $\sigma$-exceptional effective $\mathbb{Q}$-divisor $E_{\sigma}$. Thus, we have the relation

$$
\rho^{*}\left(\left.\mu^{*}\left(K_{X}+\Delta\right)\right|_{W}\right)=\rho^{*}\left(K_{W}+\left.\left(\mu_{*}^{-1} \Delta+E\right)\right|_{W}-\left.E_{\mu}\right|_{W}\right) \sim_{\mathbb{Q}} E_{\sigma}-\rho^{*}\left(\left.E_{\mu}\right|_{W}\right) .
$$

We put $E_{+}-E_{-}:=E_{\sigma}-\rho^{*}\left(\left.E_{\mu}\right|_{W}\right)$, where $E_{+}$and $E_{-}$are effective $\mathbb{Q}$-divisors that have no common irreducible components. Here, $E_{+}$is $\sigma$-exceptional.

This paragraph is due to an argument in Miyaoka [14, Proposition IV 2.4]. Put $e:=$ $\operatorname{dim} W^{\prime}$ and $c:=$ the codimension of $\sigma\left(E_{+}\right)$in $W_{l m}$. We take general members $A_{1}, A_{2}, \ldots$, $A_{e-c} \in|A|$ and $H_{1}, H_{2}, \ldots, H_{c-2} \in|H|$ where $A$ and $H$ are very ample divisors on $W_{l m}$ and $W^{\prime}$, respectively. Set

$$
S=\left(\bigcap_{i=1}^{e-c} \sigma^{-1}\left(A_{i}\right)\right) \cap\left(\bigcap_{i=1}^{c-2} H_{i}\right) .
$$

Taking into account the argument above, we proceed along the lines in [8, Proof of Theorem 7.3]. Then we have the fact that $\rho^{*}\left(\left.\mu^{*}\left(K_{X}+\Delta\right)\right|_{W}\right)$ is $\mathbb{Q}$-linearly trivial and so is $\left.\mu^{*}\left(K_{X}+\Delta\right)\right|_{W}$. From this, the assertion follows. 
In the following, we cope with the base points that lie on the non-KLT locus $\lfloor\Delta\rfloor$.

Proposition 3.2. Let $(X, \Delta)$ be a log terminal variety and $H$ a NEF $\mathbb{Q}$-Cartier $\mathbb{Q}$ divisor such that $H-\left(K_{X}+\Delta\right)$ is NEF and abundant. Assume that $v\left(X, a H-\left(K_{X}+\Delta\right)\right)=$ $v\left(X, H-\left(K_{X}+\Delta\right)\right)$ and $\kappa\left(X, a H-\left(K_{X}+\Delta\right)\right) \geq 0$ for some $a \in \mathbb{Q}$ with $a>1$. If $\left.H\right|_{[\Delta\rfloor}$ is semiample, then Bs $|m H| \cap\lfloor\Delta\rfloor=\varnothing$ for some positive integer $m$ with $m H$ being Cartier. (Here Bs $|m H|$ denotes the base locus of $|m H|$.)

Proof. From an argument in [8, Proof of Theorem 6.1] we have a diagram

$$
X \stackrel{\mu}{\longrightarrow} Y \stackrel{f}{\longrightarrow} Z
$$

with the following properties:

(a) $Y$ and $Z$ are smooth projective varieties. Moreover, $Y$ is a log resolution of $(X, \Delta)$;

(b) $\mu$ is birational and $f$ is surjective with the property that $f_{*} \mathscr{O}_{Y}=\mathscr{O}_{Z}$;

(c) $\mu^{*}\left(H-\left(K_{X}+\Delta\right)\right) \sim \mathbb{Q} f^{*} M_{0}$ for some NEF and big $\mathbb{Q}$-divisor $M_{0}$;

(d) $\mu^{*} H \sim \mathbb{Q} f^{*} H_{0}$ for some NEF $\mathbb{Q}$-divisor $H_{0}$.

We define rational numbers $a_{i}$ by $K_{Y}=\mu^{*}\left(K_{X}+\Delta\right)+\sum a_{i} E_{i}$. We may assume that $H_{0}$ and $H$ are Cartier.

We put

$$
S:=\lfloor\Delta\rfloor, \quad E:=\sum_{a_{i}>0}\left\lceil a_{i}\right\rceil E_{i}, \quad S^{\prime}:=\sum_{a_{i}=-1} E_{i}
$$

We note that, $m \mu^{*} H+E-S^{\prime}-\left(K_{Y}+\sum\left\{-a_{i}\right\} E_{i}\right)=(m-1) \mu^{*} H+\mu^{*}\left(H-\left(K_{X}+\Delta\right)\right)$, which is $\mathbb{Q}$-linearly equivalent to the inverse image of a NEF and big $\mathbb{Q}$-divisor on $Z$. There are two cases.

CASE $1\left(f\left(S^{\prime}\right) \neq Z\right)$. In this case we use Fujino's argument [3, Section 2]. By Theorem 2.3 we have an injection

$$
H^{1}\left(Y, \mathscr{O}_{Y}\left(m \mu^{*} H+E-S^{\prime}\right)\right) \longrightarrow H^{1}\left(Y, O_{Y}\left(m \mu^{*} H+E\right)\right) .
$$

Then we consider the commutative diagram

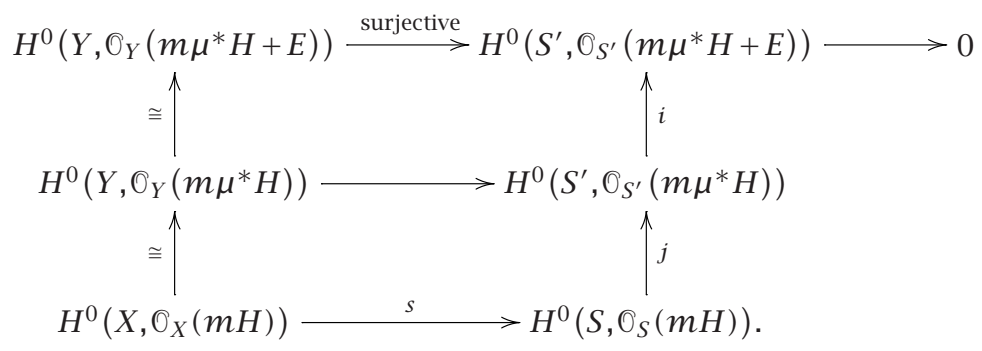

The homomorphism $i$ is injective from Lemma 2.4 and the fact that $E$ and $S^{\prime}$ have no common irreducible component. The homomorphism $j$ is injective from Lemma 2.4 and the fact that $S^{\prime} \rightarrow S$ is surjective. Thus, the homomorphism $s$ is surjective from the diagram (3.8). Consequently, $\left.|m H|\right|_{S}=|m H|_{S} \mid$. 
CASE $2\left(f\left(S^{\prime}\right)=Z\right)$. In this case we use an argument in [10, Section 7]. There exists an irreducible component $S^{\prime \prime}$ of $S^{\prime}$ such that $f\left(S^{\prime \prime}\right)=Z$. Because $\left.H\right|_{S}$ is semiample and $\mu^{*} H \sim_{\mathbb{Q}} f^{*} H_{0},\left.f^{*} H_{0}\right|_{S^{\prime \prime}}$ is semiample. Consequently, the $\mathbb{Q}$-divisor $H_{0}$ also is semiample from Lemma 2.5.

We generalize Kawamata's result [8, Theorem 6.1] (see also Theorem 1.3) concerning the semiampleness for KLT pairs to the case of log terminal pairs in the following form.

Proposition 3.3. Assume that $(X, \Delta)$ is log terminal. Let $H$ be a NEF $\mathbb{Q}$-Cartier Q-divisor on $X$ with the following properties:

(1) $H-\left(K_{X}+\Delta\right)$ is NEF and abundant;

(2) $v\left(X, a H-\left(K_{X}+\Delta\right)\right)=v\left(X, H-\left(K_{X}+\Delta\right)\right)$ and $\kappa\left(X, a H-\left(K_{X}+\Delta\right)\right) \geq 0$ for some $a \in \mathbb{Q}$ with $a>1$.

If, for some positive integer $p_{1}$, the divisor $p_{1} H$ is Cartier and $\mathrm{Bs}\left|p_{1} H\right| \cap\lfloor\Delta\rfloor=\varnothing$, then $H$ is semiample.

In the proof below we proceed along the lines in [8, Proof of Theorem 6.1] and thus omit the parts which are parallel. However, we have to be very delicate in dealing with the non-KLT locus $\lfloor\Delta\rfloor$.

Proof. From [8, Theorem 6.1], we may assume that $\lfloor\Delta\rfloor \neq 0$. Therefore, the condition that Bs $\left|p_{1} H\right| \cap\lfloor\Delta\rfloor=\varnothing$ implies that Bs $\left|p_{1} H\right| \neq X$. Thus, $\left|p_{1} t H\right| \neq \varnothing$ for all $t \in \mathbb{N}_{>0}$ (where $\mathbb{N}_{>0}$ denotes the set of all positive integers).

We have smooth projective varieties $Y$ and $Z$ and morphisms $X \stackrel{\mu}{\longrightarrow} Y \stackrel{f}{\longrightarrow} Z$ with the following properties:

(1) $\mu$ is birational and $f$ is surjective;

(2) $f_{*} \mathrm{O}_{Y}=\mathrm{O}_{Z}$;

(3) $\mu^{*}\left(H-\left(K_{X}+\Delta\right)\right) \sim \mathbb{Q} f^{*} M_{0}$ for some NEF and big $\mathbb{Q}$-divisor $M_{0}$ (where the symbol $\sim_{\mathbb{Q}}$ expresses the $\mathbb{Q}$-linear equivalence);

(4) $\mu^{*} H \sim \mathbb{Q} f^{*} H_{0}$ for some NEF $\mathbb{Q}$-divisor $H_{0}$.

We may assume that $H_{0}$ and $H$ are Cartier and $f^{*} H_{0}$ and $\mu^{*} H$ are linearly equivalent.

Putting $\Lambda(m):=\mathrm{Bs}|m H|$, we may assume that $\Lambda\left(p_{1}\right) \neq \varnothing$ (otherwise we immediately obtain the assertion). By repetition of blowing-ups over $Y$, we may replace $Y$ and get a simple normal crossing divisor $F=\sum_{i \in I} F_{i}$ on $Y$ such that

(5) $\mu^{*}\left|p_{1} H\right|=|L|+\sum_{i \in I} r_{i} F_{i}$ and $|L|$ is base point free.

Then, by replacing $Z$ and $Y$ we have $L \sim_{\mathbb{Q}} f^{*} L_{0}$ for some $\mathbb{Q}$-divisor $L_{0}$, because

$$
\begin{aligned}
v\left(Y, \mu^{*}\left(a H-\left(K_{X}+\Delta\right)\right)\right) & \geq v\left(Y,\left(\frac{(a-1)}{p_{1}}\right) L+\mu^{*}\left(H-\left(K_{X}+\Delta\right)\right)\right) \\
& \geq v\left(Y, \mu^{*}\left(H-\left(K_{X}+\Delta\right)\right)\right)
\end{aligned}
$$

from the argument in [8, Proof of Proposition 2.1]. We note that

$$
\Lambda\left(p_{1}\right)=\mu\left(\bigcup_{r_{i} \neq 0} F_{i}\right) .
$$


We have an effective divisor $M_{1}$ such that $M_{0}-\delta M_{1}$ is ample for all $\delta \in \mathbb{Q}$ with $0<\delta \ll 1$. By further repetition of blowing-ups over $Y$, we may replace $Y$ and get the following properties:

(6) $K_{Y}=\mu^{*}\left(K_{X}+\Delta\right)+\sum_{i \in I} a_{i} F_{i}$;

(7) $f^{*} M_{1}=\sum_{i \in I} b_{i} F_{i}$.

We set

$$
c:=\min _{r_{i} \neq 0} \frac{a_{i}+1-\delta b_{i}}{r_{i}} .
$$

Note that, if $a_{i}=-1$ then $\mu\left(F_{i}\right) \subset\lfloor\Delta\rfloor$ and that if $\mu\left(F_{i}\right) \subset\lfloor\Delta\rfloor$ then $r_{i}=0$ from the assumption of the theorem. Thus, by taking $\delta$ small enough, we may assume that $c>0$ and that, if $F_{i} \not \subset \mu^{-1}(\lfloor\Delta\rfloor)$, then $a_{i}+1-\delta b_{i}>0$ (even if $b_{i} \neq 0$ ). Set $I_{0}:=\left\{i \in I ; a_{i}+\right.$ $\left.1-\delta b_{i}=c r_{i}, r_{i} \neq 0\right\}$ and $\left\{Z_{\alpha}\right\}:=\left\{f(\Gamma) ; \Gamma \in \operatorname{Strata}\left(\sum_{i \in I_{0}} F_{i}\right)\right\}$. Let $Z_{1}$ be a minimal element of $\left\{Z_{\alpha}\right\}$ with respect to the inclusion relation. We note that, $Z_{1} \neq Z$. Because $M_{0}-\delta M_{1}$ is ample, for some $q \in \mathbb{N}_{>0}$, there exists a member $M_{2} \in\left|q\left(M_{0}-\delta M_{1}\right)\right|$ such that $Z_{1} \subset M_{2}$ and $Z_{\alpha} \not \subset M_{2}$ for all $\alpha \neq 1$.

We would like to show that we may assume that $\operatorname{Supp}\left(f^{*} M_{2}\right) \subset F$. Then, we investigate the variation of the numbers $a_{i}+1-\delta b_{i}$ and the set $I_{0}$ under the blowing-up $\sigma: Y^{\prime} \rightarrow Y$ with permissible smooth center $C$ with respect to $F$. We get a simple normal crossing divisor $F^{\prime}=\sum_{i \in I^{\prime}} F_{i}^{\prime}$ on $Y^{\prime}$ (where $I^{\prime}=I \cup\{0\}$ ) with the following properties:

$$
\begin{gathered}
F_{0}^{\prime}=\sigma^{-1}(C), \\
K_{Y^{\prime}}=\sigma^{*} \mu^{*}\left(K_{X}+\Delta\right)+\sum_{i \in I^{\prime}} a_{i}^{\prime} F_{i}^{\prime}, \\
\sigma^{*}\left(\sum_{i \in I} r_{i} F_{i}\right)=\sum_{i \in I^{\prime}} r_{i}^{\prime} F_{i}^{\prime}, \\
\sigma^{*} f^{*} M_{1}=\sum_{i \in I^{\prime}} b_{i}^{\prime} F_{i}^{\prime} .
\end{gathered}
$$

We set $I_{0}^{\prime}:=\left\{i \in I^{\prime} ; a_{i}^{\prime}+1-\delta b_{i}^{\prime}=c r_{i}^{\prime}, r_{i}^{\prime} \neq 0\right\}$. Let $F_{i_{1}}, \ldots, F_{i_{u}}$ be the irreducible components of $F$ that contain $C$. Let $F_{i_{j}}^{\prime}$ be the strict transform of $F_{i_{j}}$ by $\sigma$. We note that

$$
\sigma^{*}\left(K_{Y}-\sum_{j=1}^{u} a_{i_{j}} F_{i_{j}}\right)=K_{Y^{\prime}}-\left(\operatorname{codim}_{Y} C-1\right) F_{0}^{\prime}-\sum_{j=1}^{u} a_{i_{j}}\left(F_{i_{j}}^{\prime}+F_{0}^{\prime}\right) .
$$

Thus, $a_{0}^{\prime}=\left(\operatorname{codim}_{Y} C-1\right)+\sum_{j=1}^{u} a_{i_{j}}$. Therefore,

$$
a_{0}^{\prime}+1 \geq \sum_{j=1}^{u}\left(a_{i_{j}}+1\right)
$$

where the equality holds if and only if $u=\operatorname{codim}_{Y} C$. We note also that $r_{0}^{\prime}=\sum_{j=1}^{u} r_{i_{j}}$ and $b_{0}^{\prime}=\sum_{j=1}^{u} b_{i_{j}}$.

CLAIM 3.4. If $F_{0}^{\prime} \not \subset(\mu \sigma)^{-1}(\lfloor\Delta\rfloor)$, then $a_{0}^{\prime}+1-\delta b_{0}^{\prime} \geq c r_{0}^{\prime}$. The equality holds if and only if $\operatorname{codim}_{Y} C=u$ and $i_{j} \in I_{0}$ for all $j$. 
Proof of Claim 3.4. First we note the inequality

$$
a_{0}^{\prime}+1-\delta b_{0}^{\prime} \geq \sum_{j=1}^{u}\left(a_{i_{j}}+1-\delta b_{i_{j}}\right),
$$

where the equality holds if and only if $\operatorname{codim}_{Y} C=u$. Because $F_{i_{j}} \not \subset \mu^{-1}(\lfloor\Delta\rfloor)$, we have $a_{i_{j}}+1-\delta b_{i_{j}}>0$. Here if $r_{i_{j}} \neq 0$ then $a_{i_{j}}+1-\delta b_{i_{j}} \geq c r_{i_{j}}$, from the definition of $c$. On the other hand, if $r_{i_{j}}=0$ then $a_{i_{j}}+1-\delta b_{i_{j}}>c r_{i_{j}}$. Now we note the inequality

$$
\sum_{j=1}^{u}\left(a_{i_{j}}+1-\delta b_{i_{j}}\right) \geq \sum_{j=1}^{u} c r_{i_{j}},
$$

where the equality holds if and only if $r_{i_{j}} \neq 0$ and $a_{i_{j}}+1-\delta b_{i_{j}}=c r_{i_{j}}$ (i.e., $i_{j} \in I_{0}$ ) for all $j$. Here $\sum_{j=1}^{u} c r_{i_{j}}=c r_{0}^{\prime}$.

CLAIM 3.5. If $i_{j} \in I_{0}$ for all $j$ and $C \in \operatorname{Strata}\left(\sum_{j=1}^{u} F_{i_{j}}\right)$, then $I_{0}^{\prime}=I_{0} \cup\{0\}$. Otherwise, $I_{0}^{\prime}=I_{0}$.

Proof of Claim 3.5. Note that, $\operatorname{codim}_{Y} C=u$ if and only if $C \in \operatorname{Strata}\left(\sum_{j=1}^{u} F_{i_{j}}\right)$. Thus, Claim 3.4 implies the assertion, because if $F_{0}^{\prime} \subset(\mu \sigma)^{-1}(\lfloor\Delta\rfloor)$ then $r_{0}^{\prime}=0$.

ClaIm 3.6. We have an equation

$$
\min _{r_{i}^{\prime} \neq 0} \frac{a_{i}^{\prime}+1-\delta b_{i}^{\prime}}{r_{i}^{\prime}}=c .
$$

Proof of Claim 3.6. In the case where $r_{0}^{\prime} \neq 0$, we have $F_{0}^{\prime} \not \subset(\mu \sigma)^{-1}(\lfloor\Delta\rfloor)$. Thus, Claim 3.4 implies the assertion.

CLAIM 3.7. If $F_{0}^{\prime} \not \subset(\mu \sigma)^{-1}(\lfloor\Delta\rfloor)$, then $a_{0}^{\prime}+1-\delta b_{0}^{\prime}>0$.

Proof of Claim 3.7. In this case, $a_{0}^{\prime}+1>0$. If $b_{0}^{\prime} \neq 0$, then $C \subset f^{*} M_{1}$, so $u \neq 0$. Thus, $a_{0}^{\prime}+1-\delta b_{0}^{\prime} \geq \sum_{j=1}^{u}\left(a_{i_{j}}+1-\delta b_{i_{j}}\right)>0$ because all $F_{i_{j}} \not \subset \mu^{-1}(\lfloor\Delta\rfloor)$.

By virtue of Claims 3.5, 3.6, and 3.7, we may assume that $f^{*} M_{2}=\sum_{i \in I} s_{i} F_{i}$ where $F=\sum_{i \in I} F_{i}$ is a simple normal crossing divisor. We put

$$
c^{\prime}:=\min _{\mu\left(F_{i}\right) \not \subset\lfloor\Delta\rfloor} \frac{a_{i}+1-\delta b_{i}}{r_{i}+\delta^{\prime} s_{i}}
$$

and $I_{1}:=\left\{i \in I ; a_{i}+1-\delta b_{i}=c^{\prime}\left(r_{i}+\delta^{\prime} s_{i}\right), \mu\left(F_{i}\right) \not \subset\lfloor\Delta\rfloor\right\}$, for a rational number $\delta^{\prime}$ with $0<\delta^{\prime} \ll \delta$.

ClaIm 3.8. We have a relation $I_{1} \subset I_{0}$.

Proof of Claim 3.8. Because if $\mu\left(F_{i}\right) \not \subset\lfloor\Delta\rfloor$ then $a_{i}+1-\delta b_{i}>0$, in the case where $r_{i}=0$ the divisor $F_{i}$ does not attain the minimum in (3.18).

ClaIm 3.9. There exists a member $j \in I_{0}$ such that $s_{j}>0$.

Proof of Claim 3.9. The condition that $Z_{1} \subset M_{2}$ implies that, for some $j \in I$, $s_{j}>0$ and $F_{j}$ contains an element $\Gamma \in \operatorname{Strata}\left(\sum_{i \in I_{0}} F_{i}\right)$. Here $j \in I_{0}$, because $F$ is with only simple normal crossings. 
ClaIm 3.10. We have an inequality $s_{i}>0$ for all $i \in I_{1}$.

Proof OF Claim 3.10. Claims 3.8 and 3.9 and the formula (3.18) imply the assertion.

CLAIM 3.11. We have a relation $f(\Gamma)=Z_{1}$ for all $\Gamma \in \operatorname{Strata}\left(\sum_{i \in I_{1}} F_{i}\right)$.

Proof of Claim 3.11. From Claim 3.10, $f(\Gamma) \subset M_{2}$. The condition that $Z_{\alpha} \not \subset M_{2}$ for all $\alpha \neq 1$ implies the fact that $f(\Gamma) \neq Z_{\alpha}$ for all $\alpha \neq 1$. Thus, $f(\Gamma)=Z_{1}$ from Claim 3.8.

Now we set $N:=m \mu^{*} H+\sum_{i \in I}\left(-c^{\prime}\left(r_{i}+\delta^{\prime} s_{i}\right)+a_{i}-\delta b_{i}\right) F_{i}-K_{Y}$ for an integer $m \geq$ $c^{\prime} p_{1}+1$. Then,

$$
\begin{aligned}
N & =c^{\prime}\left(-\sum_{i \in I} r_{i} F_{i}\right)+m \mu^{*} H-\mu^{*}\left(K_{X}+\Delta\right)-\delta \sum_{i \in I} b_{i} F_{i}-c^{\prime} \delta^{\prime} \sum_{i \in I} s_{i} F_{i} \\
& \sim_{\mathbb{Q}} c^{\prime}\left(L-p_{1} \mu^{*} H\right)+m \mu^{*} H-\mu^{*} H+f^{*}\left(M_{0}-\delta M_{1}\right)-c^{\prime} \delta^{\prime} \sum_{i \in I} s_{i} F_{i} \\
& \sim_{\mathbb{Q}} c^{\prime} f^{*} L_{0}+\left(m-\left(c^{\prime} p_{1}+1\right)\right) \mu^{*} H+\left(1-c^{\prime} \delta^{\prime} q\right) f^{*}\left(M_{0}-\delta M_{1}\right) .
\end{aligned}
$$

Because $\mu^{*} H$ and $f^{*} H_{0}$ are linearly equivalent, $N$ is $\mathbb{Q}$-linearly equivalent to the pullback of an ample $\mathbb{Q}$-divisor on $Z$. We put

$$
\begin{aligned}
A & :=\sum_{i \in I \backslash I_{1}, \mu\left(F_{i}\right) \not\lfloor\lfloor\Delta\rfloor}\left(-c^{\prime}\left(r_{i}+\delta^{\prime} s_{i}\right)+a_{i}-\delta b_{i}\right) F_{i}, \\
B_{1} & :=\sum_{i \in I_{1}} F_{i}, \\
C & :=\sum_{\mu\left(F_{i}\right) \subset\lfloor\Delta\rfloor}\left(-c^{\prime}\left(r_{i}+\delta^{\prime} s_{i}\right)+a_{i}-\delta b_{i}\right) F_{i} .
\end{aligned}
$$

Then, $\sum_{i \in I}\left(-c^{\prime}\left(r_{i}+\delta^{\prime} s_{i}\right)+a_{i}-\delta b_{i}\right) F_{i}=A-B_{1}+C$. We express $\lceil C\rceil:=-B_{2}+B_{3}$ in effective divisors $B_{2}$ and $B_{3}$ without common irreducible components. Here, we note that $f\left(B_{1}+B_{2}\right) \neq Z$, from Claim 3.11 and from the fact that the locus $f^{-1}\left(\mathrm{Bs}\left|p_{1} H_{0}\right|\right)=$ $\mu^{-1}\left(\Lambda\left(p_{1}\right)\right) \neq \varnothing$ and the locus $\mu^{-1}(\lfloor\Delta\rfloor)$ are mutually disjoint. Note also that, $\lceil A\rceil$ and $B_{3}$ are $\mu$-exceptional effective divisors because if $a_{i}>0$ then $F_{i}$ is $\mu$-exceptional.

By Theorem 2.3, the homomorphism

$$
\begin{gathered}
H^{1}\left(Y, \mathscr{O}_{Y}\left(m \mu^{*} H+\sum_{i \in I}\left\lceil\left(-c^{\prime}\left(r_{i}+\delta^{\prime} s_{i}\right)+a_{i}-\delta b_{i}\right)\right\rceil F_{i}\right)\right) \\
\longrightarrow H^{1}\left(Y, \mathscr{O}_{Y}\left(m \mu^{*} H+\lceil A\rceil+B_{3}\right)\right)
\end{gathered}
$$

is injective because $f\left(B_{1}+B_{2}\right) \neq Z$. Hence

$$
\begin{aligned}
& H^{0}\left(Y, \bigcirc_{Y}\left(m \mu^{*} H+\lceil A\rceil+B_{3}\right)\right) \\
& \quad \longrightarrow H^{0}\left(B_{1}, \bigcirc_{B_{1}}\left(m \mu^{*} H+\lceil A\rceil+B_{3}\right)\right) \oplus H^{0}\left(B_{2}, \bigcirc_{B_{2}}\left(m \mu^{*} H+\lceil A\rceil+B_{3}\right)\right)
\end{aligned}
$$


is surjective, because $B_{1} \cap B_{2}=\varnothing$ from Claim 3.8. Here

$$
H^{0}\left(B_{1}, \mathscr{O}_{B_{1}}\left(m \mu^{*} H+\lceil A\rceil+B_{3}\right)\right) \cong H^{0}\left(B_{1}, \mathscr{O}_{B_{1}}\left(m \mu^{*} H+\lceil A\rceil\right)\right)
$$

because $B_{1} \cap B_{3}=\varnothing$ from Claim 3.8. We note that $\operatorname{Supp}\left(\left.A\right|_{B_{1}}\right)$ is with only simple normal crossings and $\left\lceil\left. A\right|_{B_{1}}\right\rceil$ is effective. Because $\left.m \mu^{*} H\right|_{B_{1}}+\left.A\right|_{B_{1}}-K_{B_{1}}=\left.N\right|_{B_{1}}$, we obtain a positive integer $p_{2}$ such that

$$
H^{0}\left(B_{1}, \mathscr{O}_{B_{1}}\left(p_{2} t \mu^{*} H+\lceil A\rceil\right)\right) \neq 0
$$

for all $t \gg 0$, from Claim 3.11 and [8, Theorem 5.1]. Consequently, the assertion of Proposition 3.3 follows.

Proof OF TheOrem 1.5. Because $\kappa\left(X, K_{X}+\Delta\right)>0$, we have $\kappa\left(X, K_{X}+\Delta\right)=$ $v\left(X, K_{X}+\Delta\right)$ from the log minimal model and the log abundance theorems in dimension less than or equal 3 (see $[10,15]$ and Proposition 3.1). We note that, $\left.\left(K_{X}+\Delta\right)\right|_{\lfloor\Delta\rfloor}$ is semiample from Proposition 2.8 and Theorem 1.6. Thus, Proposition 3.2 implies that Bs $\left|m\left(K_{X}+\Delta\right)\right| \cap\lfloor\Delta\rfloor=\varnothing$ for some $m \in \mathbb{N}_{>0}$ with $m\left(K_{X}+\Delta\right)$ being Cartier. Consequently, Proposition 3.3 gives the assertion.

ACKNOWLEDGMENT. The author would like to thank the referees for their valuable advice concerning the presentation and the quotations.

\section{REFERENCES}

[1] H. Esnault and E. Viehweg, Revêtements cycliques. II (autour du théorème d'annulation de J. Kollár), Géométrie Algébrique et Applications, II (La Rábida, 1984), Travaux en Cours, vol. 23, Hermann, Paris, 1987, pp. 81-96 (French).

[2] O. Fujino, Abundance theorem for semi log canonical threefolds, Duke Math. J. 102 (2000), no. 3, 513-532.

[3] _ Base point free theorem of Reid-Fukuda type, J. Math. Sci. Univ. Tokyo 7 (2000), no. 1, 1-5.

[4] T. Fujita, Semipositive line bundles, J. Fac. Sci. Univ. Tokyo Sect. IA Math. 30 (1983), no. 2, 353-378.

[5] _ _ Fractionally logarithmic canonical rings of algebraic surfaces, J. Fac. Sci. Univ. Tokyo Sect. IA Math. 30 (1984), no. 3, 685-696.

[6] S. Iitaka, Algebraic Geometry, Graduate Texts in Mathematics, vol. 76, Springer-Verlag, New York, 1982.

[7] Y. Kawamata, On the classification of noncomplete algebraic surfaces, Algebraic Geometry (Proc. Summer Meeting, Univ. Copenhagen, Copenhagen, 1978), Lecture Notes in Math., vol. 732, Springer, Berlin, 1979, pp. 215-232.

[8] _ Pluricanonical systems on minimal algebraic varieties, Invent. Math. 79 (1985), no. 3, 567-588.

[9] Y. Kawamata, K. Matsuda, and K. Matsuki, Introduction to the minimal model problem, Algebraic Geometry, Sendai, 1985, Adv. Stud. Pure Math., vol. 10, North-Holland Publishing, Amsterdam, 1987, pp. 283-360.

[10] S. Keel, K. Matsuki, and J. McKernan, Log abundance theorem for threefolds, Duke Math. J. 75 (1994), no. 1, 99-119.

[11] J. Kollár, Flips and abundance for algebraic threefolds, vol. 211, Société Mathématique de France, Paris, 1992.

[12] __ Shafarevich Maps and Automorphic Forms, M. B. Porter Lectures, Princeton University Press, New Jersey, 1995. 
[13] J. Kollár and S. Mori, Birational Geometry of Algebraic Varieties, Cambridge Tracts in Mathematics, vol. 134, Cambridge University Press, Cambridge, 1998.

[14] Y. Miyaoka, Geometry of rational curves on varieties, Geometry of Higher Dimensional Algebraic Varieties, Deutsche Mathematiker-Vereinigung (DMV) Seminar, vol. 26, Birkhäuser, Basel, 1997, pp. 1-127.

[15] V. V. Shokurov, 3-fold log-flips, Russian Acad. Sci. Izv. Math. 40 (1993), 95-202.

[16] E. Szabó, Divisorial log terminal singularities, J. Math. Sci. Univ. Tokyo 1 (1994), no. 3, 631-639.

SHIGETAKA FUKUDA: FACULTY OF EDUCATION, GIFU SHOTOKU GAKUEN UNIVERSITY, YANAIZUCHO, GIFU 501-6194, JAPAN

E-mail address: fukuda@ha. shotoku.ac.jp 


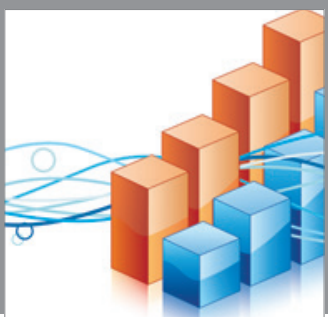

Advances in

Operations Research

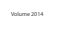

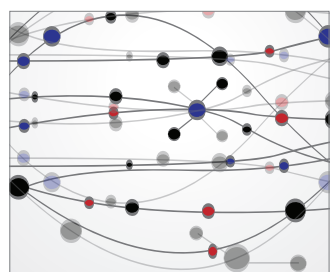

\section{The Scientific} World Journal
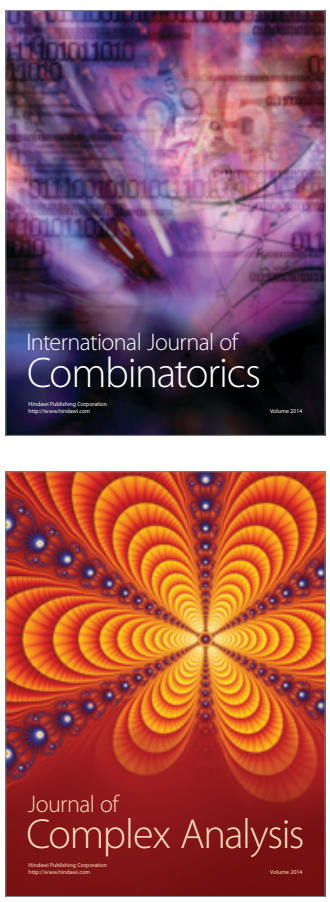

International Journal of

Mathematics and

Mathematical

Sciences
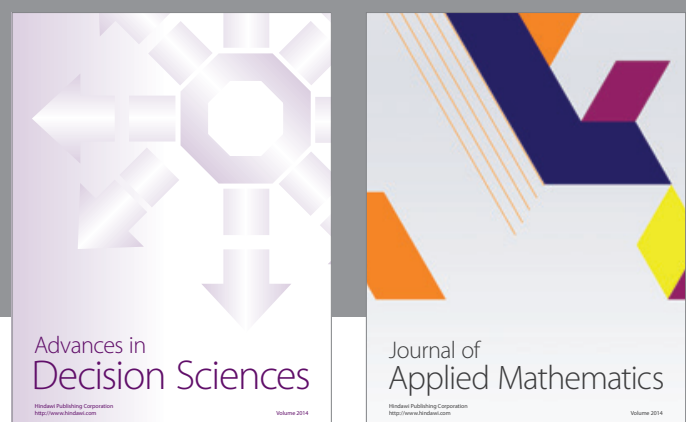

Journal of

Applied Mathematics
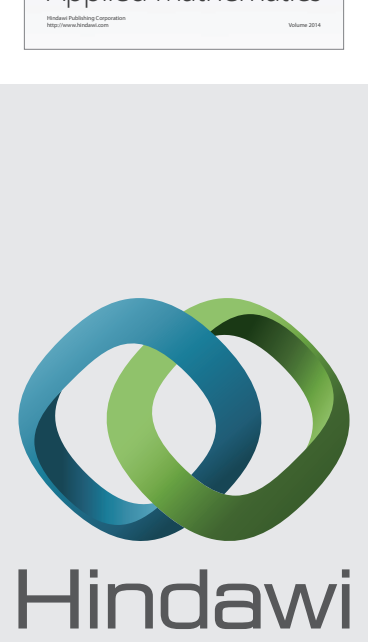

Submit your manuscripts at http://www.hindawi.com
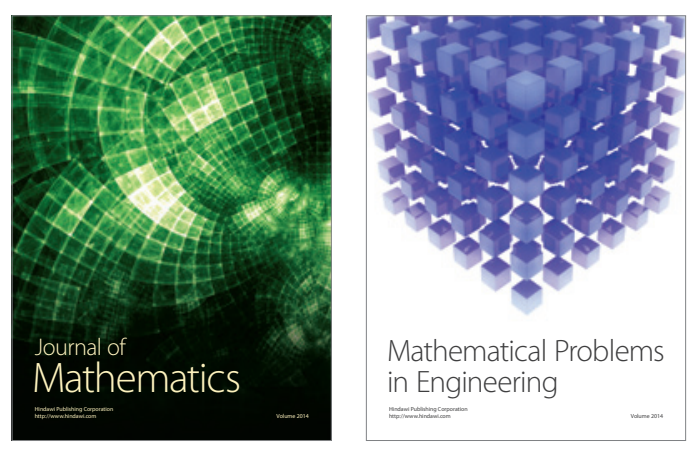

Mathematical Problems in Engineering
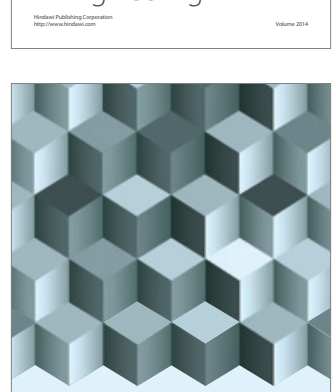

Journal of

Function Spaces
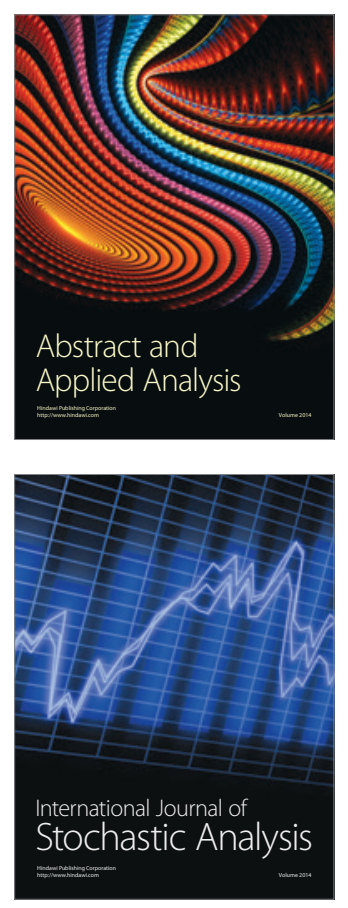

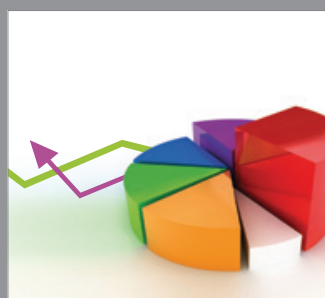

ournal of

Probability and Statistics

Promensencen
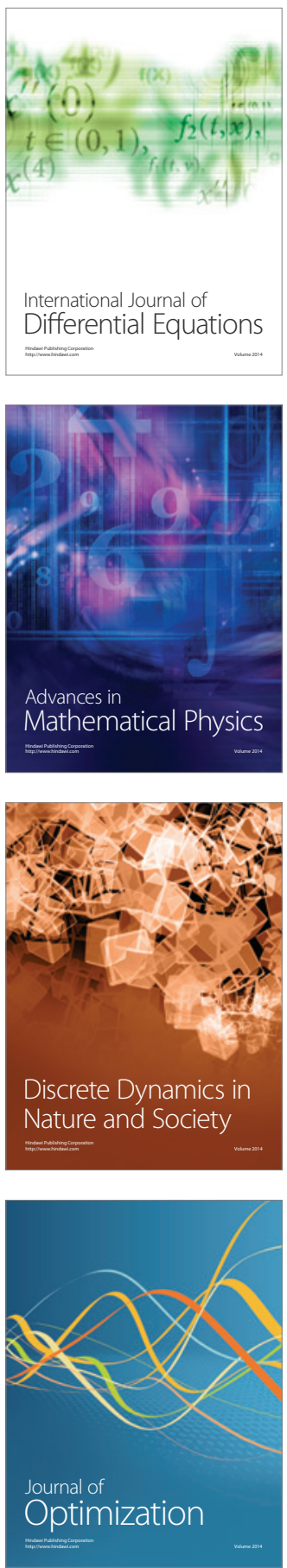